\title{
Use of a hydrophilic coating wire reduces significantly the rate of central vein punctures and the incidence of pneumothorax in totally implantable access port (TIAP) surgery
}

Georgios Polychronidis ${ }^{\dagger}$, Roland Hennes ${ }^{\dagger}$, Cosima Engerer ${ }^{1}$, Phillip Knebel ${ }^{1}$, Daniel Schultze ${ }^{1}$, Thomas Bruckner ${ }^{2}$, Beat P. Müller-Stich ${ }^{1}$ and Lars Fischer ${ }^{1,3^{*}}$

\begin{abstract}
Background: Insertion of a Totally Implantable Access Port (TIAP) can be performed either via Central Vein Puncture (CVP) or Brachiocephalic Vein Cut-down (venous section-VS). The primary success rate of TIAP implantation using VS rarely ever achieves $100 \%$. The objective of this study was to describe a modified VS approach using a hydrophilic coated wire (TVS).
\end{abstract}

Methods: From 01.01.2015 to 31.12.2015, all patients receiving TIAP implantations were screened. During this time, all patients in whom the primary VS procedure was found to be unsuccessful were analysed.

Results: In 2015, 1152 patients had TIAP implantations performed by 24 different surgeons. Of these, 277 patients needed a second line rescue strategy either by CVP $(n=69)$ or TVS $(n=208)$. There were no statistically significant differences regarding demographics or indication for TIAP implantation between CVP and TVS. The operation time and the qualification of the operating surgeon between CVP and TVS did not differ significantly. After the introduction of the guidewire with a hydrophilic coated wire, the need for CVP decreased significantly from $12.7 \%$ to $8.8 \%(p<0.0001)$. In patients receiving CVP as a second line rescue strategy, the incidence of pneumothorax ( $n=3$ patients (4.3\%)) was significantly higher compared to patients with TVS as a second line rescue strategy ( $n=1$ patient $(0.48 \%), p=0.02$ ).

Conclusion: The use of a hydrophilic coated wire significantly decreased the number of CVP and the incidence of pneumothorax. TVS is a safe and successful second-line rescue strategy.

Keywords: Totally implantable access port, Pneumothorax, Guide wire, Venous cut-down

\section{Background}

Since the first insertion of a Totally Implantable Access Port (TIAP) in 1982, the use of this central venous access system has increased dramatically over the last years [1]. As an example, the total amount of implanted TIAPs in Germany was over 125,000 in $2014[2,3]$.

\footnotetext{
* Correspondence: L.Fischer@klinikum-mittelbaden.de

${ }^{\dagger}$ Equal contributors

'Department of Surgery, University of Heidelberg, INF 110, 69120 Heidelberg, Germany

${ }^{3}$ Department of Surgery, Klinikum Mittelbaden Baden-Baden Bühl, Balger Str. 50, 76532 Baden-Baden, Germany

Full list of author information is available at the end of the article
}

Nowadays, TIAPs are not only used for chemotherapy in cases of malignant diseases, but also for blood and blood products transfusion, as well as, for the administration of parenteral nutrition [1-7].

There are two main approaches used to implant a TIAP. Firstly, via the direct puncture of a central vein (CVP) and secondly, through a classical cut-down of the brachiocephalic vein (CVS). In recent years, the CVS was adapted by using a guide-wire in a modified Seldinger technique (MVS) $[8,9]$. Both main approaches, i.e. the CVP and CVS or the MVS, can be performed with low morbidity $[10,11]$. However, the risk of pleural injury with 
consecutive development of a pneumothorax will always exist when CVP is utilised [7, 12]. On the other hand, the success rate of TIAP implantation via CVS/ MVS will never reach 100\% [10, 13-17], since there will be patients in whom CVS/MVS cannot be performed because of the absence of a brachiocephalic vein or the presence of one that is inadequate sized. Another reason for an unsuccessful TIAP implantation by CVS/ MVS is a steep junction of the brachiocephalic vein into the central venous system) [18-21].

Although the use of a guide-wire as a means of dealing with a situation in which the catheter cannot be inserted into the cephalic vein is a basic part of the MVS [22], there have not been any specifications regarding the kind of guide-wire to be used. However, clinical data derived mostly from interventional radiologist series suggest that there are guide-wires available that are either particularly suited for small vessels or able to pass through steep junctions of blood vessels [23]; the widely used Terumo ${ }^{\circ}$ Radifocus Guidewire M Standard (Terumo wire) type is one of these [24]. The Terumo ${ }^{\circ}$ wire is a guide-wire with a hydrophilic polymer coating, thus allowing for low friction manoeuvrability. The aim of this study was to examine the impact of this guide-wire (TVS) as a second line rescue strategy in patients with primary TIAP implantations.

\section{Methods}

This retrospective study was approved by the local ethics committee of the University of Heidelberg (S-584/2016). The study was conducted at the outpatient clinic of the General Surgery Department, University of Heidelberg. Funding was not provided.

\section{Participants}

The criterion for this study was to focus on all elective TIAP insertions for either benign or malignant diseases that were performed under local anaesthesia throughout 2015. A Flowchart of this retrospective study is shown in Fig. 1. Based on previously published data [19] a followup period of 6 months was established as sufficient in order to detect possible complications after TIAP

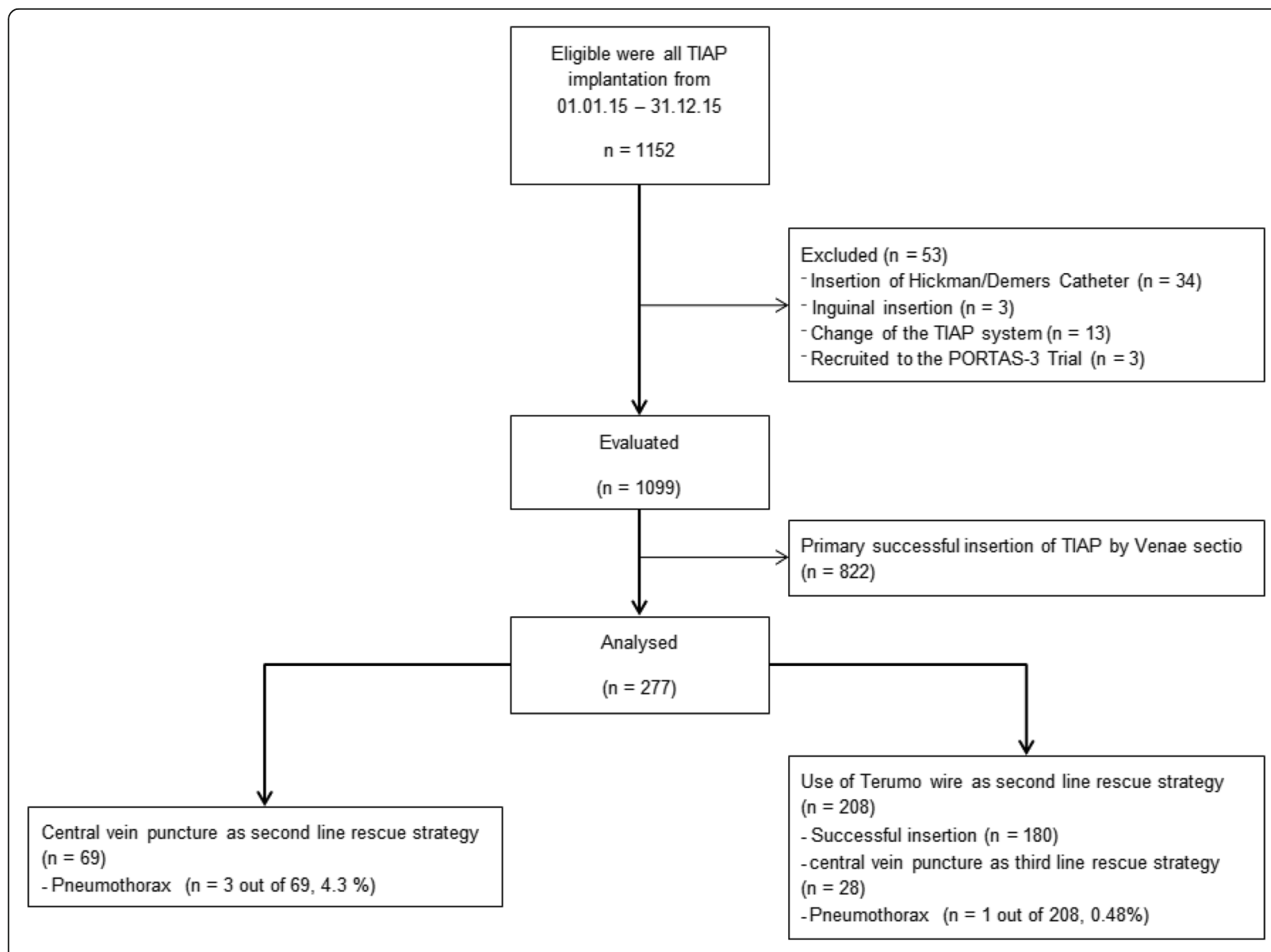

Fig. 1 Flowchart of this retrospective study 
implantation. Complications during the first 30 days after TIAP insertion were classified as early, while complications presenting after that time were classified as late complications.

\section{Interventions}

The principal techniques for CVP, CVS, and MVS, respectively, have been described in detail in previous publications $[22,25]$. The choice of the implantation side was predominantly left to the patients' discretion. In general, all patients were positioned on the operating table in a five-degree, reverse Trendelenburg's position. The neck, chest, and shoulders of the patients were prepared and draped in a sterile manner. Antibiotic prophylaxis was given in cases where patients were at risk for endocarditis (in accordance with the local standards) or only if a TIAP was to be used for chemotherapy within three days after implantation. Local anaesthesia was administered in the prospective operation area under sterile conditions and a $2-3 \mathrm{~cm}$ long skin incision was done 2-3 cm inferior of the clavicular base above the deltoidpectoral region. After exhibition of the cephalic vein, the CVS technique was evaluated.

\section{Implantation of TIAP using guidewire with a hydrophilic coating (TVS, Figs. 2a-d)}

In cases where the cephalic vein was either non-existent or too small for CVS or MVS (Fig. 2b), the guidewire with a hydrophilic coating wire was used. The TVS started with ligation of the respective vessel (typically a small cephalic vein or Ramus pectoralis that merges into the $\mathrm{V}$. thoracoacromialis) distally and encircled cranially with a 3-0 absorbable suture. With the use of magnification glasses and microsurgical instruments, a vein cutdown was performed (Fig. 2c) and the hydrophilic coated wire was inserted (Fig. 2d). Finally, the guidewire was placed under fluoroscopy to the junction of the superior Vena Cava and right atrium. After positioning, either the TIAP catheter or a vein dilator and sheath were passed over the guide-wire. In cases where the latter was performed, the guide-wire and dilator were removed and the catheter was introduced through the peel-away sheath. After removal of the peel-away sheath, correct positioning of the implant was once again checked via fluoroscopy. Subsequently, the steps for port chamber connection and port chamber placement were done as per in-house standards (as described in previous publications) [22]. After CVP a routine $\mathrm{x}$-ray was performed postoperatively to evaluate for pneumothorax.

\section{Objectives and outcomes}

The primary aim was to establish whether or not the use of a guidewire with a hydrophilic coating reduced the number of punctures needed without correspondingly increasing complication rates. Also, the aim was to establish that neither the patients' characteristics nor the surgeons' experience play any significant role in the successful use of this guide-wire. Secondary aims of this
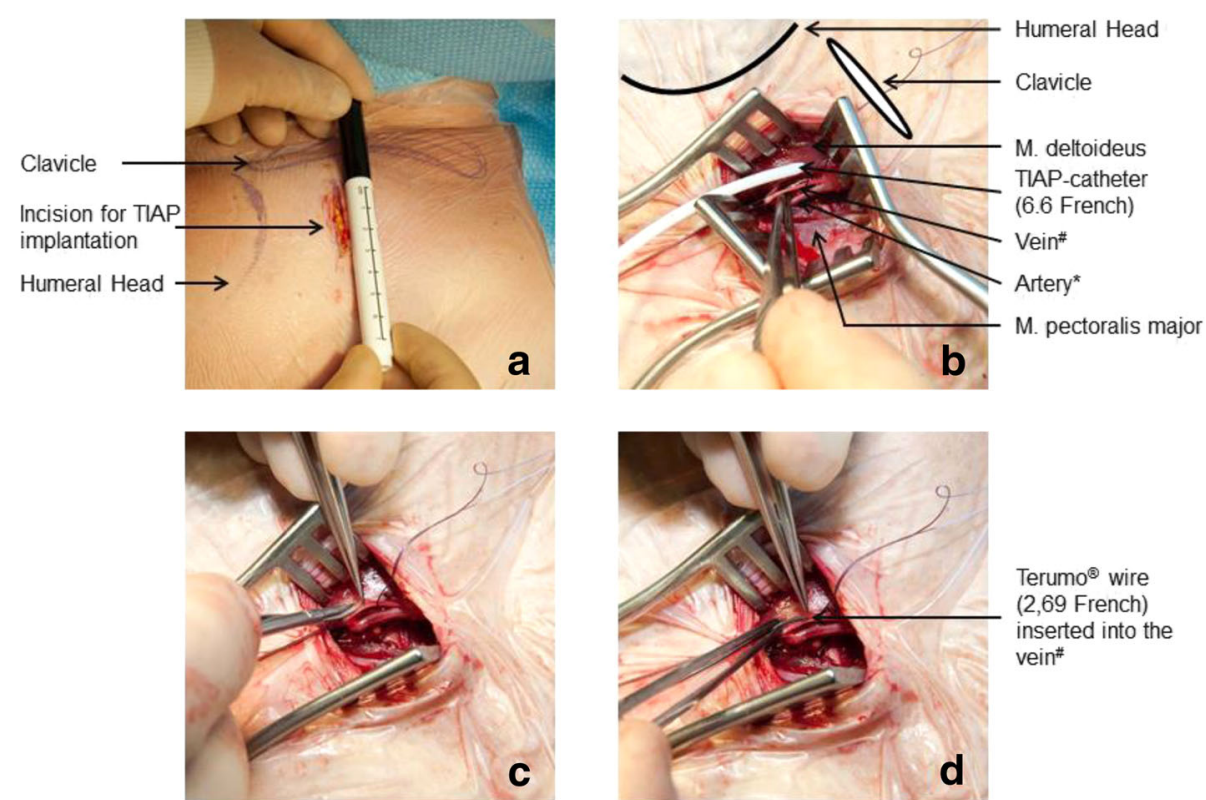

Fig. 2 a-d Incision for Totally Implantable Access Port (TIAP) implantation according to: anatomical landmarks (a), with intraoperative situs (b), Vein cut-down/Nenae Sectio using microsurgical instruments (c), and insertion of the guidewire with a hydrophilic coating (Terumo ${ }^{\circledR}$ wire) (d). \# Ramus pectoralis merging into $V$. thoracoacromialis. * Ramus pectoralis of A. thoracoacromialis 
study were to establish the independence of the results in cases where the operation was performed by an attending or a resident physician.

\section{Statistical analysis}

The complication rates and the baseline characteristics were analysed with respect to the technique which was actually applied in each case. The success rate in the various treatment groups was determined and subsequently compared using the Kruskal-Wallis Test for continuous variables (Standard Deviation is provided in Tables 1 and 2) and Chi-square for dichotomous variables.

The rates of complications per year were calculated using a two-tailed chi-squared test. All analyses were performed using SPSS version 21.

\section{Results}

In 2015, 1152 patients underwent operations that were classified as 'Insertions of Intravenous Devices'. Overall, TIAP implantations were performed by 24 different surgeons, 9 of whom were board certified (data not shown). Out of the 1152 patients, 1099 patients actually received TIAP implantations, of which, the TIAP implantations were successfully performed via classical vein cut-down (CVS) in 822 cases (74.8\%). Some 277 patients needed a second line rescue strategy, either via a primary Central Vein Puncture (CVP) or a modified vein cut-down using the hydrophilic coated guide-wire (TVS, Fig. 1 and Table 1). There were no statistically significant differences regarding demographics or indication for TIAP implantation (Table 1, $p>0.1, p$ - values not shown) between CVP and TVS.

The operation time and the qualification of the operating surgeon between CVP and TVS showed no significant differences (Table 2). It was found that the use of the hydrophilic coated guide-wire as a second line rescue strategy reduced the need for CVP significantly from $12.7 \%$ to $8.8 \%(p<0.0001$, Fig. 3$)$. Regarding early morbidity the incidence of pneumothorax was significantly higher in patients receiving CVP as a second line rescue strategy when compared to patients with TVS as a second line rescue strategy ( $p=0.02$, Table 2$)$. There were no differences between CVP and TVS (Table 2) as it relates to late morbidity after TIAP implantation; this includes infection, non-function, and/or removal of TIAP.

\section{Discussion}

\section{Safety of the TVS strategy}

The data presented on the 277 patients who needed a second line rescue therapy during TIAP implantation allows the conclusion, that the use of the hydrophilic coated guide-wire as a second line rescue strategy significantly reduces not only the puncture rate during TIAP implantation, but also the incidence of pneumothorax. It is true that in experienced hands TIAP implantations can be performed safely whether or not CVP or CVS with its modifications are being used. Even though CVP includes a risk, per se, of developing of a pneumothorax, the published data reports this complication in about $1-3.2 \%$ of all cases [10, 26-28]. The reason to use primarily CVS and its modifications at our department is based on the fact that we generally perform more than 1000 TIAP implantations per year. For example, this means that even with a low, $3 \%$ incidence rate of pneumothorax after CVP, 30 of our patients would experience such complications on average. Furthermore, TIAP implantations are considered a reliable teaching procedure and will mostly be performed by residents during the early course of their training $[27,29]$.

As stated before, TIAP implantation using CVS (and its modifications) is not successful in all patients. It has been shown, under conditions of a randomized

Table 1 Baseline Characteristics of Analysed Patients

\begin{tabular}{|c|c|c|c|}
\hline & All Patients n (\%) & $\begin{array}{l}\text { Central Vein Puncture as a } \\
\text { second line rescue strategy n (\%) }\end{array}$ & $\begin{array}{l}\text { Use of guidewire with a hydrophilic coating } \\
\text { as a second line rescue strategy } n(\%)\end{array}$ \\
\hline$n$ & 277 & 69 & 208 \\
\hline Mean Age & 58.7 years $(S D \pm 15.1)$ & 58.6 years $(S D \pm 16.4)$ & 58.7 years $(S D \pm 15.1)$ \\
\hline Female Patients & $149(53.7 \%)$ & $38(55.1 \%)$ & $111(53.4 \%)$ \\
\hline \multicolumn{4}{|l|}{ Indication for TIAP Implantation } \\
\hline Gastrointestinal Malignancy & $84(30.3 \%)$ & $19(27.5 \%)$ & $65(31.2 \%)$ \\
\hline Gynecological Malignancy & $74(26.7 \%)$ & $20(29.0 \%)$ & $54(26.0 \%)$ \\
\hline Hematological Malignancy & $59(21.3 \%)$ & $15(21.7 \%)$ & $44(21.2 \%)$ \\
\hline Other Malignancies/Reasons & $60(21.7 \%)$ & $15(21.7 \%)$ & $45(21.6 \%)$ \\
\hline Patients with first TIAP implantation & $245(88.4 \%)$ & $58(84.1 \%)$ & $187(89.9 \%)$ \\
\hline TIAP implantation on the patients' left side & $220(79.4 \%)$ & $58(84.1 \%)$ & $163(78.4 \%)$ \\
\hline Central Vein Puncture & $100(36.1 \%)$ & $69(100 \%)$ & $31(14.9 \%)$ \\
\hline
\end{tabular}


Table 2 Intra- and postoperative data of Totally Implantable Access Port (TIAP) implantation using either Central Vein Puncture (CVP) or modified vein cut-down using hydrophilic coated guide-wire (TVS) as a second line rescue strategy

\begin{tabular}{|c|c|c|c|c|}
\hline & All Patients $\mathrm{n}(\%)$ & $\begin{array}{l}\text { Central Vein Puncture as a } \\
\text { second line rescue strategy } n(\%)\end{array}$ & $\begin{array}{l}\text { Use of guidewire with a hydrophilic } \\
\text { coating as a second line strategy } n(\%)\end{array}$ & $p^{*}$ \\
\hline$n$ & 277 & 69 & 208 & \\
\hline Mean operation time (SD) & $\begin{array}{l}37.7 \mathrm{~min} \\
( \pm 20.9 \mathrm{~min})\end{array}$ & $\begin{array}{l}35.6 \min \\
( \pm 16.8 \mathrm{~min})\end{array}$ & $\begin{array}{l}38.4 \min \\
( \pm 22.2 \mathrm{~min})\end{array}$ & 0.347 \\
\hline Operation performed by Resident alone (n) & $76(27.5 \%)$ & $16(23.2 \%)$ & $60(28.8 \%)$ & 0.351 \\
\hline Operation performed by Attending alone ( $\mathrm{n}$ ) & $171(61.7 \%)$ & $45(65.2 \%)$ & $126(60.6 \%)$ & 0.568 \\
\hline $\begin{array}{l}\text { Operation performed by Resident and } \\
\text { Attending }\end{array}$ & $30(10.8 \%)$ & $8(11.6 \%)$ & $22(10.6 \%)$ & 0.814 \\
\hline \multicolumn{5}{|l|}{ Early Morbidity } \\
\hline Intraoperative bleeding & $3(1 \%)$ & $0(0 \%)$ & $3(1 \%)$ & 0.316 \\
\hline Pneumothorax & $4(1.4 \%)$ & $3(4.3 \%)$ & $1(0.5 \%)$ & 0.020 \\
\hline Need for thorax drainage & $1(0.4 \%)$ & $1(1.4 \%)$ & $0(0 \%)$ & 0.082 \\
\hline \multicolumn{5}{|l|}{ Late Morbidity } \\
\hline Thrombosis & $2(0.7 \%)$ & $0(0 \%)$ & $2(0.9 \%)$ & 0.414 \\
\hline TIAP Infection & $17(6.1 \%)$ & $3(1.4 \%)$ & $14(6.7 \%)$ & 0.475 \\
\hline Sepsis due to TIAP Infection & $14(5 \%)$ & $2(2.9 \%)$ & $12(5.7 \%)$ & 0.346 \\
\hline Non-Function due to dislocation & $8(2.9 \%)$ & $2(2.9 \%)$ & $6(2.9 \%)$ & 0.995 \\
\hline Pinch-off syndrome & $1(0.4 \%)$ & $0(0 \%)$ & $1(0.5 \%)$ & 0.564 \\
\hline Postoperative hematoma & $2(0.7 \%)$ & $0(0 \%)$ & $2(0.9 \%)$ & 0.414 \\
\hline Removal of TIAP & $26(9.4 \%)$ & $6(8.7 \%)$ & $20(9.6 \%)$ & 0.803 \\
\hline
\end{tabular}

Abbreviations: $\mathrm{SD}=$ standard deviation, TIAP = Totally Implantable Access Port, $\mathrm{CVP}=$ Central Vein Puncture, TVS = vein cut down using a guidewire with a hydrophilic coating; Terumo ${ }^{\circledR}$ Venae Sectio

${ }^{*} p$ value determined according to the Kruskal Wallis Test for continuous variables and Chi-squared Test for dichotomous variables

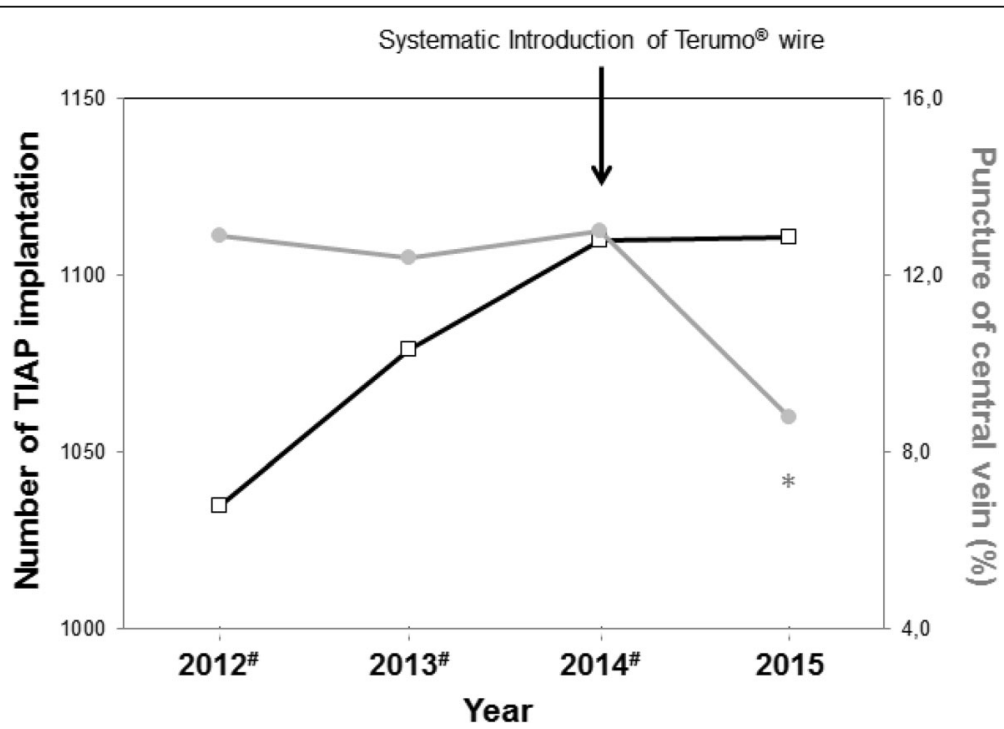

Fig. 3 Number of Totally Implantable Access Port (TIAP) implantations (black boxes) and percentage of performed Central Vein Punctures (CVP) (grey circles) performed between 2012 and 2015. * The two-tailed $p$ value is less than 0.0001 (as seen in a comparison of 2015 with 2014). \# Up to December 2013, modified vein cut-down was performed by using a MHMedical Tec $\mathrm{GmbH}^{\circledast}$ guide-wire. The Terumo ${ }^{\oplus}$ wire was used sporadically in individual patients throughout 2014; however, it was introduced systematically in 2015 
controlled trial, that TIAP implantation can be performed successfully in $92 \%$ of patients by using CVS [22]. However, the circumstances for TIAP implantation in our study could best be described as "daily routine". Overall the frequency of complications is comparable to the existing literature and much lower in the case of thrombosis, suboptimal positioning or frequency of removal [29-32]. There has been a -not statistically significant- increased rate of infections in the study group which could not be due to the slightly increased operation time. According to an analysis conducted in our centre [19] some other reasons can account for this: ongoing chemotherapy, the female gender and breast cancer were recognized as independent and significant parameters. In the study group, there are more female patients with gynaecological malignancies, which could explain this finding.

Retrospectively seen, the need for CVP averaged about $12 \%$ throughout the years in our centre. This, together with the fact that an increasing number of patients with a need for recurrent TIAP implantation and patients with inadequately sized or even non-existent brachiocephalic vein, increases the need for appropriate and applicable modification techniques for CVS.

\section{Reproducibility and learning curve of the TVS strategy} At first glance, the process of TIAP implantation using TVS (as described in Fig. 1) seems technically demanding. However, the presented data has shown that the operation time for TIAP implantation between CVP and TVS do not differ significantly. In addition, $28.8 \%$ of the residents were able to apply this technique safely by themselves, while only $10.6 \%$ of cases required the support of an attending physician. This makes our findings, that TVS reduces significantly not only the puncture rate but also the incidence of pneumothorax even more clinically valuable. With this in mind, it would be interesting to examine the success and complication rates between CVP and SVS, for instance, in a multicentre expert-based setting.

\section{Conclusion}

TIAP implantation using a guidewire with a hydrophilic coating significantly reduces the need for central vein puncture and the incidence of pneumothorax. TVS can be safely applied by surgeons at any stage of their training. Subsequently, it may even be beneficial to consider TVS as a first line technique for TIAP implantation.

\section{Abbreviations}

CVP: Direct puncture of a central vein; CVS: Cut-down of the brachiocephalic vein; MVS: modified Seldinger technique (MVS); TIAP: Totally Implantable Access Port; TVS: Terumo ${ }^{\oplus}$ Guide-wire as a second line rescue strategy

\section{Acknowledgements}

The authors would like to thank Mrs. Nadya Philipps for providing with the necessary corrections as a native speaker.

\section{Funding}

We acknowledge financial support of the publication costs by Deutsche Forschungsgemeinschaft and Ruprecht-Karls-Universität Heidelberg within the funding program Open Access Publishing.

\section{Availability of data and materials}

The datasets generated and/or analysed during the current study are not publicly available due to initial approval of the ethics commission (stating that the datasets will be saved in an anonymous form and will be provided after publication on request) but are available from the corresponding author on reasonable request.

\section{Authors' contributions}

LF, GP, RH, DS developed the study idea, CE and GP acquired patient data. GP and TB performed the statistical analysis. LF, GP, BPMS and PK drafted the first version of the article. All authors contributed significantly to finalize the article and approved the definitive version.

\section{Ethics approval and consent to participate}

This retrospective study was approved by the local ethics committee of the University of Heidelberg (S-584/2016).

\section{Consent for publication}

Not applicable

\section{Competing interests}

The authors declare no competing interests.

\section{Publisher's Note}

Springer Nature remains neutral with regard to jurisdictional claims in published maps and institutional affiliations.

\section{Author details}

${ }^{1}$ Department of Surgery, University of Heidelberg, INF 110, 69120 Heidelberg, Germany. ${ }^{2}$ Department of Medical Biometry, Institute of Medical Biometry and Informatics (IMBI), Im Neuenheimer Feld 130.3, 69120 Heidelberg, Germany. ${ }^{3}$ Department of Surgery, Klinikum Mittelbaden Baden-Baden Bühl, Balger Str. 50, 76532 Baden-Baden, Germany.

Received: 16 July 2017 Accepted: 27 November 2017

Published online: 07 December 2017

\section{References}

1. Niederhuber JE, et al. Totally implanted venous and arterial access system to replace external catheters in cancer treatment. Surgery. 1982;92(4):706-12.

2. Rotzinger $\mathrm{R}$, et al. Placement of central venous port catheters and peripherally inserted central catheters in the routine clinical setting of a radiology department: analysis of costs and intervention duration learning curve. Acta Radiol. 2017:284185117695664

3. Bramesfeld A, et al. Development of quality indicators and data assessment strategies for the prevention of central venous catheter-related bloodstream infections (CRBSI). BMC Infect Dis. 2015;15(1):435

4. Adler A, et al. Infectious complications of implantable ports and Hickman catheters in paediatric haematology-oncology patients. J Hosp Infect. 2006; 62(3):358-65.

5. $\mathrm{Ng} \mathrm{F}$, et al. A comparison of Hickman line- and port-a-Cath-associated complications in patients with solid tumours undergoing chemotherapy. Clin Oncol (R Coll Radiol). 2007;19(7):551-6.

6. Vegunta RK, et al. Differences in the outcome of surgically placed long-term central venous catheters in neonates: neck vs groin placement. J Pediatr Surg. 2005:40(1):47-51.

7. Mirro J, et al. Paper presented at the 20th annual meeting of the American pediatric surgical association part 1A comparison of placement techniques and complications of externalized catheters and implantable port use in children with cancer. J Pediatr Surg. 1990;25(1):120-4.

8. Chuter T, Starker PM. Placement of Hickman-Broviac catheters in the cephalic vein. Surg Gynecol Obstet. 1988;166(2):163-4. 
9. Au FC. The anatomy of the cephalic vein. Am Surg. 1989;55(10):638-9.

10. Seiler $\mathrm{CM}$, et al. Surgical technique for totally implantable access ports (TIAP) needs improvement: a multivariate analysis of 400 patients. J Surg Oncol. 2006;93(1):24-9.

11. Knebel P, et al. Comparison of venae Sectio vs. modified Seldinger technique for totally implantable access ports; Portas-trial [ISRCTN: 52368201]. Trials. 2006;7:20.

12. Klaiber U, et al. Closed cannulation of subclavian vein vs open cut-down of cephalic vein for totally implantable venous access port (TVAP) implantation: protocol for a systematic review and proportional meta-analysis of perioperative and postoperative complications. Syst Rev. 2015;4:53.

13. Davis SJ, Thompson JS, Edney JA. Insertion of Hickman catheters. A comparison of cutdown and percutaneous techniques. Am Surg. 1984; 50(12):673-6.

14. Perry EP, Nash JR, Klidjian AM. Direct cephalic vein cannulation for safe subclavian access. J R Coll Surg Edinb. 1990;35(4):218-20.

15. Torramade JR, et al. The complications of central venous access systems: a study of 218 patients. Eur J Surg. 1993;159(6-7):323-7.

16. Gallichio MH, et al. Placement of a double lumen silastic catheter for hemodialysis access through the cephalic vein. J Am Coll Surg. 1994; 178(2):171-2.

17. Povoski SP. A prospective analysis of the cephalic vein cutdown approach for chronic indwelling central venous access in 100 consecutive cancer patients. Ann Surg Oncol. 2000;7(7):496-502.

18. Bowdle $A$, et al. Ultrasound identification of the guidewire in the brachiocephalic vein for the prevention of inadvertent arterial catheterization during internal jugular central venous catheter placement. Anesth Analg. 2016;123(4):896-900.

19. Fischer $L$, et al. Reasons for explantation of totally implantable access ports: a multivariate analysis of 385 consecutive patients. Ann Surg Oncol. 2008; 15(4):1124-9.

20. Hickman RO, et al. A modified right atrial catheter for access to the venous system in marrow transplant recipients. Surg Gynecol Obstet. 1979;148(6): $871-5$.

21. Broviac JW, Cole JJ, Scribner BH. A silicone rubber atrial catheter for prolonged parenteral alimentation. Surg Gynecol Obstet. 1973;136(4):602-6.

22. Knebel $\mathrm{P}$, et al. Protocol of an expertise based randomized trial comparing surgical Venae Sectio versus radiological puncture of Vena Subclavia for insertion of Totally Implantable Access Port in oncological patients. Trials. 2008;9:60.

23. Kahler J, et al. Initial experience with a hydrophilic-coated guidewire for recanalization of chronic coronary occlusions. Catheter Cardiovasc Interv. 2000;49(1):45-50.

24. Gray DF, et al. Balloon angioplasty of totally and subtotally occluded coronary arteries: results using the Hydrophillic Terumo Radifocus guidewire M (glidewire). Catheter Cardiovasc Diagn. 1993;30(4):293-9.

25. Huttner FJ, et al. Frequency of pneumothorax and haemothorax after primary open versus closed implantation strategies for insertion of a totally implantable venous access port in oncological patients: study protocol for a randomised controlled trial. Trials. 2015;16:128.

26. Di Carlo I, et al. Totally implantable venous access devices implanted surgically: a retrospective study on early and late complications. Arch Surg. 2001;136(9):1050-3.

27. Fosh, B., M. Canepa, and M. Eaton, Long-term venous access insertion: 'the learning curve'. ANZ Journal of Surgery, 2015: p. n/a-n/a.

28. Feo CF, et al. Cost and morbidity analysis of chest port insertion in adults: outpatient clinic versus operating room placement. Annals of Medicine and Surgery. 2017;21:81-4.

29. Voog E, et al. Totally implantable venous access ports: a prospective longterm study of early and late complications in adult patients with cancer. Support Care Cancer. 2017;

30. Wang YC, et al. Long-term outcomes of totally implantable venous access devices. Support Care Cancer. 2017;25(7):2049-54.

31. Biffi R, et al. Cost effectiveness of different central venous approaches for port placement and use in adult oncology patients: evidence from a randomized three-arm trial. Ann Surg Oncol. 2014;21(12):3725-31.

32. Tabatabaie $\mathrm{O}$, et al. Upper extremity deep venous thrombosis after port insertion: what are the risk factors? Surgery. 2017:162(2):437-44.

\section{Submit your next manuscript to BioMed Central and we will help you at every step:}

- We accept pre-submission inquiries

- Our selector tool helps you to find the most relevant journal

- We provide round the clock customer support

- Convenient online submission

- Thorough peer review

- Inclusion in PubMed and all major indexing services

- Maximum visibility for your research

Submit your manuscript at www.biomedcentral.com/submit
Biomed Central 